\title{
Plan Local de Convivencia de El Molar: Una Experiencia de Participación Ciudadana en la Mancomunidad de Servicios Sociales Vega del Guadalix
}

\section{Coexistence Local Plan of El Molar: An Experience of Citizenship Participation in the Social Services Community of the Vega del Guadalix}

\author{
Fernando Sabín Galán y Nuria Sánchez Díaz \\ Andaira Investigación e Intervención Social
}

\author{
Equipo de Servicios Sociales \\ de la Mancomunidad Vega de Guadalix
}

\begin{abstract}
Resumen. El Plan Local de Convivencia del municipio de El Molar (Madrid) es una experiencia de participación ciudadana que lleva en marcha desde 2005. Este plan tiene un doble objetivo. Por un lado, mejorar la convivencia en el municipio entendiendo que el origen de las personas es una diferencia más a tener en cuenta y por tanto los esfuerzos deben dirigirse a "desactivar" esa frontera invisible que aparece entre un "ellos" (extranjeros) y un "nosotros" (autóctonos). Por otro lado, se persigue normalizar el acceso a los recursos por parte de toda la población molareña, adecuando los servicios municipales a la diversidad de sus habitantes (teniendo en cuenta el origen de las personas, pero también otras diferencias relevantes como el género, la edad, la clase social,...).

Para el desarrollo de este proceso se vienen utilizando metodologías participativas basadas en la realización de un diagnóstico de la situación del municipio (fase de análisis de necesidades y demandas comunes), la búsqueda de soluciones colectivas (fase de recogida y debate de propuestas) y, la puesta en marcha de las mismas (fase actual de implementación de las medidas del Plan).
\end{abstract}

Palabras clave: participación, convivencia, diversidad, normalización.

Summary. The Local Plan for the improvement of the coexistence between the inhabitants of the El Molar municipality (Madrid) is a citizenship participation experience that has been developed since 2005. This plan has a double objective. On the one hand, to improve the coexistence in the municipality understanding that the origin of the people is a difference more to take in consideration, and therefore the efforts must go "to desactivate" that invisible border that appears between "they" (foreigners) and "us" (natives). On the other hand, it aims to standardize resources access to all the municipality's population, adapting the municipal services to the diversity of its inhabitants (considering the origin of the people, but also other important differences like gender, age, social class,...).

For the development of this process, participatory methodologies are being used in order to accomplish a diagnosis of the municipality's situation (analytic phase of necessities and common demands), the search for collective solutions (proposals phase for the collection and debate of ideas) and, the start-up (present phase for the measures implementation).

Key words: participation, coexistence, diversity, normalization.

La correspondencia sobre este artículo dirigirla a: Andaira investigación e intervención social S.Coop.Mad. C/ Montera 9 - 3º - 28013

Madrid. E-mail: cooperativa@andaira.net 


\section{Introducción}

En abril de 2005 se comenzaron a dar los primeros pasos para la puesta en marcha del Plan Local de Convivencia del municipio de El Molar. Este proyecto, financiado por la Consejería de Inmigración de la Comunidad de Madrid, ha sido promovido en un primer momento desde la Concejalía de Servicios Sociales del municipio a través de su área técnica y en un segundo momento por la Mancomunidad de Servicios Sociales "Vega del Guadalix"1, contando durante todo el proceso con Andaira S. Coop. Mad, cooperativa especialista en investigación e intervención social, para la dinamización del proceso.

La finalidad de este Plan no es únicamente "integrar a las personas inmigrantes extranjeras", sino poner en marcha instrumentos que contribuyan a construir un municipio para todos los vecinos y vecinas que conviven en El Molar. Esto, indudablemente, pasa por desarrollar instrumentos para "integrar" a la población inmigrante, en el sentido de facilitar su equiparación en el acceso a recursos, derechos y obligaciones con respecto al resto de la ciudadanía, favoreciendo así su implicación en la construcción de la sociedad molareña y contribuyendo a consolidar su sentido de pertenencia a la misma. De la misma manera, supone desarrollar herramientas para impulsar y mejorar la convivencia dirigidas a todos los vecinos y vecinas de El Molar, a través de las cuales se sensibilice, se favorezca el conocimiento mutuo, se saque partido a la oportunidad que supone una sociedad más diversa, se facilite la participación que hace posible la corresponsabilidad en la construcción de la misma, se luche contra las diferentes formas de discriminación y se contribuya a prevenir y gestionar de manera positiva los conflictos que también forman parte de la convivencia.

Para garantizar la sostenibilidad del Plan se ha creído oportuno y necesario contar con la participación de las personas que habitan el municipio, verdaderas protagonistas de su día a día así como de su futuro. Por este motivo, tanto el diseño como la puesta en marcha del propio Plan parten de un pro-

1 La Mancomunidad de Servicios Sociales "Vega del Guadalix" fue constituida el 14 de marzo de 2007 y se compone de la unión de los municipios: El Molar, Guadalix de la Sierra, Pedrezuela y San agustín de Guadalix. ceso de participación ciudadana que busca garantizar la escucha de los intereses y demandas de toda la población y favorecer su implicación en la búsqueda de soluciones.

La base central del Plan Local de Convivencia está asentada en la creación de dos espacios de trabajo que han permitido involucrar a diferentes actores a lo largo de todo el proceso: la Comisión de Seguimiento (formada por entidades sociales, representantes políticos y personal técnico de la administración) y la Mesa de Convivencia (compuesta por el tejido asociativo y vecinos y vecinas de El Molar).

A través de estos espacios se ha articulado todo el proceso de participación donde cabe distinguir tres momentos principales: fase de análisis y diagnóstico, fase de recogida de propuestas y priorización de las líneas principales del Plan, y puesta en marcha de las diferentes actuaciones.

Actualmente, este Plan continúa desarrollándose con la implementación de las medidas y propuestas surgidas del proceso. Las experiencias acumuladas a lo largo de estos 3 años, fruto de la participación de multitud de personas, asociaciones e instituciones, han permitido la confección de este artículo, y a todos ellos agradecemos desde aquí su esfuerzo y dedicación.

\section{Contexto de la intervención: El Molar en cifras}

El Molar ha sufrido una gran transformación demográfica y económica en los últimos años. El fuerte crecimiento económico de la región madrileña en el último decenio ha tenido un gran impacto sobre las estructuras productivas de municipios, como El Molar, históricamente más alejados de las dinámicas económicas regionales. Estos municipios se han visto inmersos dentro de la gran ola urbanizadora estableciéndose la construcción como principal fuente de ingresos municipales y principal factor de creación de empleo. Este proceso ha supuesto un incremento significativo de la población atraída por un mercado inmobiliario más asequible para la economía familiar.

Así mismo, este auge en el sector de la construcción ha concentrado una gran cantidad de mano de obra atrayendo a población extranjera en busca de tra- 
bajo. En el Molar, según los últimos datos disponibles del censo del 2001, el porcentaje de personas ocupadas en el sector de la construcción era del $16,95 \%$ cuando en la Comunidad de Madrid en ese mismo año el porcentaje era del 9,61\%. Otro dato que ejemplifica la importancia que está adoptando este sector se refiere al número de personas ocupadas que trabajan en actividades inmobiliarias y de alquiler por cada 1.000 habitantes situado en un $13,36 \%$, porcentaje superado sólo por la hostelería y el comercio.

El Molar, un municipio del que tradicionalmente ha emigrado población joven a la capital en busca de trabajo, se ha convertido en los últimos 10 años en municipio receptor de inmigración. Este aumento de su población ha hecho que se duplique el número de habitantes pasando de 3.400 habitantes en 1996 a cerca de 7.000 a comienzos de 2008.

El Molar es un municipio que ha experimentado un crecimiento vegetativo de su población en los últimos años gracias a la aportación de población extranjera, ya que su población española se ha reducido. Una parte importante de estos nuevos vecinos y vecinas son de origen extranjero, cifra que ha ido aumentando paulatinamente en los últimos años predominando ligeramente la presencia masculina dentro de este colectivo. Actualmente, la población inmigrante en el municipio es de $1.834^{2}$ personas ( $28,4 \%$ de la población total).

La composición de dicha población por áreas de procedencia y peso demográfico es la siguiente: países de Europa del Este (Bulgaria y Rumanía), Latinoamérica (Ecuador y Colombia) y Marruecos ${ }^{3}$ El Molar es, por tanto, un municipio que ha experimentado en pocos años un importante incremento de personas de origen extranjero que ha rejuvenecido la población al tiempo que ha incrementado la diversidad de origen de sus habitantes.

\section{¿Por qué construir un Plan Local de Convivencia participativo? entre lo común y lo diverso}

La práctica nos demuestra día a día que el principal instrumento para el cambio social son las perso-

\footnotetext{
2 Observatorio Regional de Inmigración. Datos de octubre 2008.

${ }^{3}$ Datos facilitados por el "Plan Local de Convivencia de el Molar,
}

nas. La llegada a un determinado territorio de personas procedentes de otros lugares no hace más que incidir en este tipo de procesos acelerando la tendencia al cambio por el mero contacto con otras formas de vida. En un tema, como es el de la convivencia, una intervención en la comunidad y desde la propia comunidad es esencial ya que estamos hablando de cambios en valores, actitudes, formas de relacionarse, etc.

Ante este complejo panorama es necesario preguntarse hacia dónde queremos que se dirijan estos cambios. Construir un plan colectivo que dé respuesta al tipo de cambios que son necesarios para mejorar la convivencia en un territorio determinado es un ejercicio necesario en la medida en que, si ese plan colectivo no existe, y no se empieza a construir, corremos el peligro de que se impongan de manera explícita o implícita los intereses particulares de una de las partes.

En este sentido, nuestros esfuerzos han ido dirigidos a construir un plan que responda a los intereses y necesidades de todas las personas que conviven en un mismo territorio. Cuando hablamos de todas las personas, nos referimos a que los protagonistas del plan deben ser tanto la población inmigrante como la "sociedad de acogida", ya que los cambios deben ser en todas direcciones y con el esfuerzo de todas las partes. Igualmente, es importante insistir en que estas transformaciones sólo son posibles desde la práctica colectiva siendo uno de nuestros retos lograr que estas nuevas formas de hacer y relacionarse se den conjuntamente entre el ámbito institucional, técnico y ciudadano.

Ante la alteridad desde la que se suele tratar el fenómeno de la inmigración y la convivencia (dicotomía autóctono-inmigrante), hemos visto necesario elaborar este plan desde lo que nos une, desde lo que nos atraviesa, lo que denominamos "lo común". Lo común, no como una categoría estanca, sino como espacio a construir. Un camino poco transitado pero necesario (porque para convivir necesitamos pactar unos mínimos) y al mismo tiempo imposible (porque ante la complejidad que entraña este ejercicio, hay momentos en los que se aleja totalmente de la posibilidad factible). Ante esta pareja de términos el sociólogo J. Ibáñez incita a "cambiar las reglas del juego", a establecer la conversación en otras coor- 
denadas que no sean las un "ellos" y un "nosotros" (Ibáñez, 1994).

Es importante no confundir ese común con el intento de homogeneización propio de muchos enfoques científicos en el estudio y análisis de la diversidad. Más arriba insistíamos en que el papel protagonista de un proceso de este tipo lo deben llevar tanto la población inmigrante como la sociedad de acogida. Es importante matizar este punto de partida ya que la llegada al municipio de personas procedentes de diferentes lugares no es su única fuente de diversidad. El Molar era ya muy diverso antes de la llegada de población inmigrante si tenemos en cuenta otras diferencias de género, de edad, de clase social, de orientación sexual...

Por este motivo, entendemos que para el desarrollo de procesos de convivencia, el origen de las personas es sólo una diferencia más a tener en cuenta. De lo contrario, corremos el riesgo de reforzar los límites que existen entre los diferentes grupos sociales desviando la atención de lo que tienen en común, encubriendo el carácter cada vez más polícromo y polimorfo de los propios grupos y sobredeterminando variables culturales.

De ahí que uno de nuestros principales objetivos sea abrirnos a la complejidad en lugar de eludirla tratando de "desactivar" esa frontera que aparece entre un "ellos" y un "nosotros" ya que entendemos que hay muchos ellos y muchos nosotros posibles.

Para lograr "desactivar" esta frontera hemos diferenciado dos dimensiones estratégicas sobre las que trabajar colectivamente: la de los problemas o síntomas (diagnóstico de la situación del municipio) y la de las propuestas (programación de las medidas y actuaciones a desarrollar). Se trata de dos dimensiones que nos ayudan a construir ese común: por un lado, la dimensión de los problemas, que parte de necesidades comunes y no de la pertenencia a un grupo, y, por otro lado, la búsqueda de soluciones colectivas a los mismos partiendo de intereses comunes.

\section{Tres años de Plan}

Trabajar estas dos dimensiones (problemas compartidos y soluciones colectivas) tratando de involu- crar a diferentes personas y colectivos no es una tarea fácil. Requiere una metodología participativa que parta de un análisis de la realidad abriendo un proceso donde se generan acuerdos de acción consensuados dirigidos a transformar la realidad en aquellos ámbitos susceptibles de ser mejorados en tanto que producen situaciones insatisfactorias para la población (Villasante, Montañés y Martí, 2000). El reto es involucrar a la población a lo largo de todo este proceso: desde la formulación y definición del objeto de estudio hasta la discusión sobre cómo encontrar soluciones y programar y realizar propuestas (Villasante y Martín, 2001).

A continuación explicamos cómo se han abordado estas dimensiones a lo largo de estos tres años en los que el Plan ha atravesado diferentes fases diferenciadas cronológicamente:

1. Negociación de la demanda y difusión del Plan

2. Diagnóstico de la realidad social

3. Elaboración y priorización de propuestas

4. Implementación y consolidación

\section{Fase 0: Negociación de la demanda y difusión del Plan}

$\mathrm{El}$ aterrizaje de un equipo técnico a un territorio con la intención de intervenir sobre el statu quo existente y transformar colectivamente los valores, actitudes y formas de relacionarse de su población no es nada sencillo. Hay que diseñar escrupulosamente los primeros pasos que se dan porque van a marcar de forma determinante la capacidad del equipo para convertirse una herramienta útil por parte de los ámbitos institucional, técnico y ciudadano del municipio. En lo que Manuel Montañés llama conversación o reflexividad inicial (Montañés, 2006), se definen dos aspectos fundamentales de la primera fase de desarrollo de un proyecto de estas características:

- Difusión del proyecto.

- Redefinición del punto de partida.

Siguiendo la línea que marcaban los primeros planes de inmigración y la propia nomenclatura que utiliza la Comunidad de Madrid en los convenios que firma con los municipios, la Concejalía de Servicios Sociales del municipio convoca un concurso para la realización de un Plan "para" las personas inmigran- 
tes, entendidas éstas como un colectivo más menos uniforme e identificable. El por qué de un Plan de Inmigración venía justificado por la situación sociodemográfica que hemos descrito anteriormente. Estamos ante un municipio que tiene que afrontar una rápida escalada en la complejidad social a todos los niveles, con las oportunidades y amenazas que supone esto. Son muchos los términos que entran en juego: acogida, integración, interculturalidad, diversidad, asimilación... pero sólo es una palabra la que va a adjetivar al Plan y en este caso se había elegido "inmigración".

La carta de presentación que tiene el equipo es mostrar las bondades de un proyecto incipiente, pero sobre todo escuchar activamente al personal técnico y a la vecindad acerca del municipio y su realidad social mediante la realización de entrevistas grupales y personales semi-estructuradas. Es en estas primeras conversaciones con el personal técnico y representantes del tejido asociativo (mayoritariamente población de origen español) donde surgen varias reflexiones que motivan un cambio sobre el enfoque de partida del proyecto. De manera sintética se valoró que el Plan de Inmigración:

- se vinculaba a nuevas atribuciones de privilegios hacia la población inmigrante que se sumaban a las ya consolidadas en el imaginario colectivo.

- Surgen quejas acerca de la inadecuación de los recursos públicos básicos (sanidad, educación,...) al creciente volumen de población del municipio. Este hecho aumentaba la percepción de que, ante problemas comunes, se focalizaba la ayuda sobre un sector de la población.

- Se siente que iniciativas de este tipo aumentan la ya existente barrera simbólica entre el "nosotros" (personas autóctonas de origen nacional) y el "ellos" (población de origen extranjero)

Tras varias reuniones informales con vecinos y vecinas (pertenecientes mayoritariamente al tejido asociativo) y personal técnico municipal, se llegó a la conclusión de que para lograr integrar desde el inicio las diferentes perspectivas e inquietudes acerca de cómo se quería caminar hacia una mejora de la cohesión social del las personas residentes en el municipio, teniendo en cuenta la realidad que supone que un $27 \%$ de la población sea de origen extran- jero, era necesario partir de un enfoque más amplio que pudiera involucrar de la misma manera a todos los vecinos y vecinas del municipio.

Finalmente, se decidió sustituir el concepto "Inmigración" por el de "Convivencia", cambiando al mismo tiempo todo el enfoque de la intervención. De esta manera, el proyecto no nació siendo percibido como excluyente y generó un mayor interés tanto en el nivel político como en el social. Desde este nuevo enfoque se buscaba contribuir a la construcción de la sociedad de El Molar sobre la base de la convivencia para lograr una ciudadanía cohesionada capaz de trabajar conjuntamente por su presente y su futuro.

En definitiva, cuando se habla de convivencia, se hace referencia a la vida que comparten individuos, familias y grupos en cuanto a intereses, inquietudes, problemas, soluciones a dichos problemas, expectativas, usos del espacio, servicios y todo aquello que forma parte de la vida en sociedad. La convivencia implica, por lo tanto, estar al mismo tiempo y en el mismo lugar que otras personas, con las que se interactúa activa y creativamente, con quienes se comparten aspectos comunes, y entre quienes se da un entendimiento, una empatía. Sin embargo, convivir no significa estar de acuerdo en todo, sino la posibilidad de disentir, debatir y regular ese conflicto sin que ello suponga una ruptura, una desintegración o la pérdida de cohesión social.

\section{Fase 1: Diagnóstico de la realidad social}

La fase 0 coincide en parte con los primeros pasos del análisis de la realidad. A través de diferentes técnicas metodológicas comenzamos a elaborar un diagnóstico haciendo énfasis en las necesidades, los conflictos latentes y manifiestos, las oportunidades existentes en el territorio, las fortalezas, así como la percepción que se tenía de los cambios que se venían produciendo en el municipio (del lado de la vecindad autóctona) y la percepción sobre el proceso de acogida (del lado de la nueva vecindad).

Un paso previo al inicio del trabajo de campo per se consistió en la recogida de información que incluye la recopilación y análisis de información general y específica de los diferentes colectivos con los que 
se va a realizar el proyecto, así como del territorio concreto en el que se está desarrollando la investigación. Esto nos permitió contextualizar y contrastar el conocimiento que se fue produciendo a lo largo del proceso con las informaciones y los datos existentes, así como disponer de las características de la población para la construcción de muestras teóricas en el diseño de técnicas cualitativas, especialmente las entrevistas.

La primera fase del trabajo de campo consistió principalmente en la realización de entrevistas semiestructuradas a representantes políticos, personal técnico y representantes del tejido asociativo. Estas entrevistas nos aportan un conocimiento sobre los discursos que se producen y reproducen desde distintas posiciones sociales en torno a: identidades e imágenes asociadas a personas, grupos e instituciones, y relaciones que se establecen entre los mismos puntos de vista en torno a la problemática abordada; lógicas de acción, proyectos, estrategias explicitas e implícitas; estructuras de sentido, valores y normas.

Para complementar la información recogida a través de las entrevistas individuales, también se desarrollaron entrevistas grupales a vecinos y vecinas con el fin de obtener diferentes discursos sobre las diversas representaciones colectivas, así como las estructuras grupales que se articulan en torno a la problemática social que estamos estudiando.

Además, el trabajo de campo ha incluido observación participante lo cual nos permitió, al mismo tiempo que participábamos de diferentes situaciones, analizar la realidad en la que estábamos interactuando. Dentro de este tipo de observaciones cabe destacar:

- Visitas a "colectivos" o asociaciones informales $\mathrm{y}$ a asociaciones formales que nos han permitido conocer cuales son sus demandas y sus intereses, sus debilidades y sus fortalezas. Igualmente es necesario participar de estos espacios para crear y recrear redes y para la construcción de propuestas comunes que favorezcan la resolución de la problemática a resolver.

- Participación en encuentros, que nos han permitido acercarnos a la realidad y formar parte de ella. Así hemos conseguido una mayor comunicación con nuestros sujetos de estudio ampliando nuestros conocimientos acerca del contexto en el que nos encontramos.

- Transectos o paseos guiados como forma de ir hilando las situaciones y experiencias que componen los recorridos cotidianos de los diferentes colectivos que conviven en el municipio. Optamos por recorrer los espacios cotidianos de cada cual (calles, casas, empresas, transporte, supermercados, bares, comercios, etc.) como si se tratase de una entrevista en movimiento aprovechando las conversaciones que surgen por el camino.

También se optó por el uso de metodologías cuantitativas a través de la realización de una encuesta sobre percepciones, prejuicios y oportunidades de la convivencia.

De forma paralela, y con la intención de iniciar el proceso participativo, se realizaron diferentes talleres de creatividad social para la recogida de información a partir de la reflexión grupal.

\section{Fase 2: Elaboración y priorización de propuestas}

Una vez finalizado el estudio de la realidad social se diseñaron dos sesiones de devolución de resultados con la Comisión de Seguimiento y la Mesa de Convivencia que daban paso a la fase de recogida de propuestas para la elaboración final del Plan Local de Convivencia.

Estos dos grupos de trabajo, la Comisión de Seguimiento y la Mesa de Convivencia, se crearon en paralelo al diagnóstico y han permitido la recogida de diferentes propuestas en esta fase así como la participación institucional, técnica y ciudadana a lo largo de todo el proceso.

Por un lado, la Comisión de Seguimiento que, como señalábamos al comienzo, es un grupo que reúne al equipo investigador, entidades sociales, representantes políticos y personal técnico de la administración (policía, servicios sociales, empleo y desarrollo local, educación y juventud) con el objetivo de debatir, valorar y realizar el seguimiento de cada una de las fases de la investigación que ha tenido como resultado este Plan, así como su puesta en marcha, desarrollo y evaluación.

Copyright 2009 by the Colegio Oficial de Psicólogos de Madrid ISSN: 1132-0559 
Por otro lado, la Mesa de Convivencia (compuesta por el tejido asociativo, vecinos y vecinas de El Molar y el equipo investigador), cuyas funciones son realizar un seguimiento cotidiano y una discusión crítica del diagnóstico; plantear y debatir los puntos de vista de las y los representantes institucionales y asociativos; difundir el Plan, e incorporar a nuevos miembros, así como elaborar y debatir propuestas.

En estos espacios de trabajo se han consensuado los objetivos del Plan, así como sus ámbitos de actuación, permitiendo debatir y priorizar medidas y acciones concretas. El final de la fase culminó con la aprobación del Plan en el Pleno Municipal y la presentación pública y difusión del mismo.

\section{Fase 3: Implementación y consolidación del Plan}

Una vez detectadas las diferentes inquietudes y cohesionados los grupos de trabajo, se pasa a implementar el Plan con la puesta en marcha de diferentes actividades y medidas en ámbitos diversos como infancia y juventud, servicios sociales, salud, empleo, educación, etc. Se trata de dotar al Plan de un enfoque global, puesto que la convivencia es un proceso que afecta a todas las facetas de la vida cívica, social, económica, cultural y política de las personas de los grupos sociales.

Todas estas medidas responden a dos objetivos estratégicos:

- La adecuación de los recursos a la diversidad así como la normalización de los mismos.

- La mejora de la convivencia a través de la participación como clave para la construcción de una ciudadanía inclusiva.

Partimos de la idea de que las políticas municipales se dirigen al conjunto de la ciudadanía y que las actuaciones públicas deben ir orientadas a promover y garantizar el acceso normalizado de la población inmigrante a los servicios públicos y privados de carácter general. Este principio de universalidad implica que las personas inmigrantes deben recibir una atención normalizada dentro de esos servicios, aunque pueden existir algunas fases del proceso de migratorio (como la enseñanza del idioma) que pueden exigir intervenciones o servicios específicos en algunos campos, al menos con carácter temporal.

En paralelo a esta idea, consideramos que el fomento de la participación y el trabajo en red es uno de los pilares fundamentales para la puesta en marcha y el desarrollo de este Plan. La diversidad de ámbitos de trabajo y actuaciones que contempla el Plan permite dar cabida a una participación más amplia de la población basada en las inquietudes y necesidades que tienen las personas, colectivos $\mathrm{u}$ organizaciones, independientemente de su lugar de origen. La participación activa de la población molareña en todos los ámbitos sociales y en los asuntos públicos es el elemento clave para hablar propiamente de una sociedad inclusiva, cohesionada y capaz de generar un sentimiento de pertenencia en todos los vecinos y vecinas. De ahí que el grado de participación social se convierta en una de las claves que permite medir el grado éxito de la sociedad molareña a la hora de integrar a sus nuevos vecinos $\mathrm{y}$ vecinas.

\section{Logros y límites del Plan Local de Convivencia}

Actualmente el Plan continúa su desarrollo implementando medidas que nacen de un proceso participativo que lleva un recorrido de tres años. A lo largo de este proceso se ha llegado a la convicción de que todos los esfuerzos tanto a nivel ciudadano como técnico y político deben perseguir la normalización de la diversidad, entendiendo que la diversidad es intrínseca a todas las personas y colectivos. Esta diversidad es fruto de múltiples factores, entre los que se puede encontrar el lugar de origen, motivo por el cual este factor no puede determinar el acceso a determinadas actuaciones o servicios municipales y actividades comunitarias.

Pero todavía quedan múltiples retos como es el de tratar de formular intervenciones que luchen contra las múltiples discriminaciones que se dan en las vidas individuales y colectivas. Se trata de incidir sobre la interacción simultánea de múltiples diferencias humanas según género, origen, clase, religión, orientación sexual, edad, estatus de ciudadanía o condiciones de salud. Es importante considerar la coherencia institucional a la hora de emprender este tipo de procesos participativos. 
Intervenciones que abordan la discriminación de género, por un lado, o el racismo, por el otro, pero que no toman en cuenta la realidad de las personas afectadas por un conjunto de múltiples discriminaciones, difícilmente pueden dar lugar a respuestas integrales. En ese sentido, la tradicional sectorialidad de la administración pública tanto a nivel local como regional o nacional supone un fuerte hándicap a esta coherencia.

Todo esto nos lleva a concluir la necesidad de evitar la creación de jerarquías entre las diferentes formas de discriminación. No se trata de compartimentalizar las políticas, sino de formular estrategias para desafiarlas conjuntamente apoyándonos en un análisis común de cómo se conectan y articulan. Cuanto mayor es la complejidad de los problemas y mayores son las incertidumbres, más necesario es contar con la ciudadanía y los diferentes actores institucionales. Será en el marco de estas redes de participación donde podremos generar más capacidad para construir acuerdos, conocer la realidad en la que nos movemos y adoptar soluciones que se adapten mejor a las necesidades sociales.

No obstante, la participación ciudadana en la práctica presenta limitaciones importantes. En ese sentido, la cuestión radica en cómo pensar procesos de participación con una legitimidad ampliamente reconocida que al mismo tiempo respondan a los requerimientos de eficacia marcados desde las administraciones que financian estos procesos. Hay resultados que son intangibles pero no por ello son menos importantes, como puede ser el caso de la generación de una cultura cívica participativa entre la ciudadanía, la sociedad civil, la administración pública y los representantes políticos. Se trata de un difícil equilibrio entre los ritmos de la emergencia y los ritmos de un proceso a largo plazo que realmente contribuya a generar identidades compartidas y una visión integral e interrelacionada de los problemas y oportunidades que se abordan, así como una perspectiva a futuro que permita comprender la dinámica a largo plazo de las cuestiones.

\section{Bibliografía}

Ibáñez, J. (1994). Por una sociología de la vida cotidiana. México: $\mathrm{s}$. XXI.

Montañés, M. (2006). Diseño de una estrategia participativa conversacional con la que propiciar la formulación participada del Plan Local de Inmigración. En M. Montañés y J. Encina (Coordinadores) Construyendo colectivamente la convivencia en la diversidad. (165-207). Sevilla: Atrapasueños/Unilco

Villasante, T., Montañés M. y Martí J. (Eds) (2000). La investigación social participativa. Construyendo ciudadanía 1. Barcelona: El Viejo Topo.

Villasante, T. y Martín, P. (Eds) (2001). Prácticas locales de creatividad social. Construyendo ciudadanía 2. Barcelona: El Viejo Topo.

Manuscrito recibido: 23/01/2009

Revisión recibida: 04/03/2009

Manuscrito aceptado: 16/03/2009 